\title{
Numerical Simulation of Combustible Liquid Behavior and Temperature Distribution in a Solid- liquid Mixed Layer during Flame Spread over It
}

\author{
KAZUNORI KUWANA, MASATARO SUZUKI, \\ RITSU DOBASHI and TOSHISUKE HIRANO \\ Department of Chemical System Engineering \\ Faculty of Engineering \\ The University of Tokyo \\ Tokyo, Japan
}

\begin{abstract}
A numerical study has been performed of combustible liquid behavior and temperature distribution in a solid-liquid mixed layer during flame spread over it when the main mode of heat transfer is convection.

Heat transfer to the layer ahead of the leading flame edge through the solid-liquid mixed layer is shown to occur mainly near the layer surface. It is found that the liquid motion ahead of the leading flame edge is induced by a combined force of surface tension and buoyancy. This implies the importance of buoyancy to understand the flame spread over a liquid soaked in porous solid. The predicted temperature distributions fairly well coincide with the experimental results, and it is found that the spread rate $U$ can be approximately determined by selecting $U$ which gives an appropriate value of the heat transfer from gas phase to the solid-liquid layer beneath the leading flame edge.
\end{abstract}

KEY WORDS: Flame Spread; Liquid Soaked Solid Bed; Numerical Simulation; Oil Fire

\section{INTRODUCTION}

In a case when combustible liquid is accidentally spilled, it most likely soaks into a porous solid such as soil, mat or carpet. If a fire occurs, a flame will spread over the porous solid soaked with a combustible liquid. The characteristics of the flame spread phenomena in such a case are extremely different from those of the flame spread over a free-surface liquid pool. Main causes of such differences are attributable to the behavior of liquid[1,2]. Thus, it is important to 
Takeno and Hirano performed experimental studies[1, 2] of the flame spread over glass beads soaked with n-decane (flash point: $46^{\circ} \mathrm{C}$ ), whose initial temperature is $23^{\circ} \mathrm{C}$, and the flame spread rate $U$ was found to depend significantly on the diameter $d$ of solid particle[2]. For an initial liquid temperature much lower than its flash point, preheating of the liquid ahead of the leading flame edge is needed for flame spreading, since the concentration of combustible vapor ahead of the leading flame edge is not sufficient for burning. This preheating process must significantly affect the flame behavior. In the case to be examined in this study, it can be considered that the heat transfer to the liquid ahead of the leading flame edge mainly occurs through the solid-liquid mixed layer.

In the study of Takeno and Hirano, they attempted to explore the mechanisms of heat transfer process through the solid-liquid mixed layer[2]. For small solid particles, a liquid flow was scarcely induced and the main mode of heat transfer was indicated to be conduction through the solid-liquid mixed layer. The aspects of flame spread in this case were clearly explained on the basis of conductive heat transfer. For large solid particles, on the other hand, a liquid flow was observed and the main mode of heat transfer was assumed to be convection. This assumption was attempted to be verified by changing the viscosity of the liquid[2]. However, this verification is not sufficient to explain the aspects of flame spread because of complexity of the induced flow field and resulting heat transfer process. There still remain a lot of ambiguities in the flame spread process in this case. Thus, in the present study a numerical simulation of liquid behavior and temperature distribution in a solid-liquid mixed layer during flame spread over it has been performed to understand the mechanisms of flame spread when the main mode of heat transfer is convection.

\section{FORMULATION OF PROBLEM}

\section{Model}

For the flame spread over a free-surface liquid pool, some numerical studies were performed[3-7]. The results of these numerical simulations agreed well with those of experiments, and gave us a lot of knowledge. In the present study, we adopt some assumptions in these studies.

Since the purpose of the present study is to predict liquid behavior and temperature distribution in a solid-liquid mixed layer, the influence of gas phase is simulated by prescribing thermal boundary conditions at the surface of the solid-liquid mixed layer, as adopted by Torrance[3], and Torrance and Mahajan[4,5] for numerical simulations of flame spread over a liquid pool.

In this study, the conservation equations for the solid-liquid mixed layer are based on a general flow model which regards the effects of the solid particles as the frictional force proportional to the liquid velocity and as the effective thermal diffusivity of the mixed layer, and the flow and temperature fields are resolved.

Figure 1(a) shows a schematic of the physical model for the present numerical simulation. Solid 
particles are of a uniform diameter $d$, so that the solid-liquid mixed layer is uniform. The flame moves at a steady speed $U$ which is a flame spread rate. The bottom boundary is assumed to be a rigid, no-slip surface at a constant temperature $T_{0}$. Also, The surface temperature $T_{1}$ beneath the flame is constant. At the interface between the gas phase and solid-liquid mixed layer ahead of the leading flame edge, heat transfer must be much smaller than that beneath the flame. Therefore, the layer surface is assumed to be adiabatic ahead of the flame[3-5]. Far downstream of the leading flame edge, fully developed flow is assumed.

The solid-liquid mixed layer is assumed to be of a constant depth $h$. For the flame spread over a solid-liquid mixed layer, dry or gas-liquid coexisting region was observed and the thickness of this region was proportional to $d^{-1}[1,2]$. Therefore, it can be valid for a large particle diameter to assume the depth of the mixed layer as constant.

The constant properties of solid particles and liquid are assumed. Also, the Boussinesq approximation is employed, that is, the liquid density is assumed constant except in the term of buoyancy. For the simulation of the liquid flow, the Boussinesq approximation was often employed[3-7]. Although the effects of variable properties can be important for advanced predictions, it is reasonable to assume constant properties for solid particles and liquid, because confirmation of controlling mechanisms is the first purpose of this study.

For the convenience of the analysis, the coordinates are fixed at the leading flame cdge as shown in Fig. 1(b), so that the solid-liquid layer moves at a velocity of $U$ in the direction opposed to the flame spread, and the flow is assumed to be steady. Major assumptions adopted in the present study on the basis of the above discussion are summarized as follows:

1) The flow is steady and two-dimensional.

2) The surface temperature is constant beneath the flame, and the layer surfacc is adiabatic ahead of the flame.

3) Fuel vaporization is negligible, and the depth of the solid-liquid mixed layer is constant.

4) The properties of solid particles and liquid are constant.

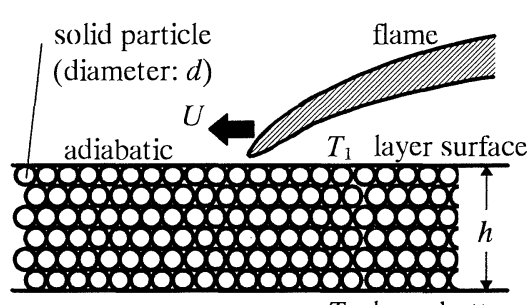

$T_{0}$ layer bottom

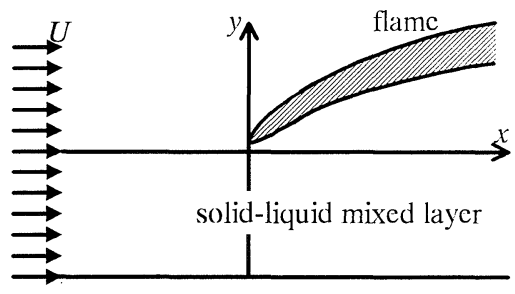

(b)

(a)

FIGURE 1 Schematics of the model adopted in the present study, (a) physical model, $T_{1}$ : temperature at the layer surface beneath the flame, $T_{0}$ : temperaturc at the bottom of the mixed layer, $U$ : flame spread rate, $d$ : diameter of solid particle, $h$ : depth of the mixed layer, (b) coordinates fixed at the leading flame edge. 
5) Liquid density is constant except for variations induced by buoyancy described according to the Boussinesq approximation.

\section{Basic Equations}

For a two-dimensional field of Boussinesq liquid, the basic equations can be stated in terms of vorticity $\omega$, stream function $\psi$, and temperature $T$. For the convenience of the analysis, the following non-dimensional quantities are introduced.

$$
\begin{aligned}
& x^{\prime}=\frac{x}{h}, y^{\prime}=\frac{y}{h}, u_{x}^{\prime}=\frac{h}{\kappa_{\mathrm{eff}}} u_{x}, u_{y}^{\prime}=\frac{h}{\kappa_{\mathrm{eff}}} u_{y}, U^{\prime}=\frac{h}{\kappa_{\mathrm{eff}}} U, \\
& d^{\prime}=\frac{d}{h}, T^{\prime}=\frac{T-T_{0}}{T_{1}-T_{0}}, \psi^{\prime}=\frac{\psi}{\kappa_{\mathrm{eff}}}, \omega^{\prime}=\frac{h^{2}}{\kappa_{\mathrm{eff}}} \omega,
\end{aligned}
$$

where $\kappa_{\text {eff }}$ denotes the effective thermal diffusivity of the solid-liquid mixed layer. The effective thermal diffusivity is given as

$\kappa_{\text {eff }}=\frac{\lambda_{\text {eff }}}{\varepsilon \rho_{1} c_{1}}=\frac{\varepsilon \lambda_{1}+(1-\varepsilon) \lambda_{\mathrm{s}}}{\varepsilon \rho_{1} c_{1}}$,

where $\lambda, c$, and $\varepsilon$ denote the thermal conductivity, specific heat, and porosity, respectively, and subscript $1, \mathrm{~s}$, and eff denote the liquid, solid and effective, respectively. When solid particles are packed most closely, porosity $\varepsilon$, independently of particle diameter, is constant $(\varepsilon=0.26)$. Further, since the properties of liquid and solid particles are assumed to be constant in this study(Assumption 4)), $\kappa_{\text {eff }}$ is constant throughout the present study.

The stream function and vorticity definitions yield

$$
\begin{aligned}
& u_{x}^{\prime}=-\frac{\partial \psi^{\prime}}{\partial y^{\prime}}, u_{y}^{\prime}=\frac{\partial \psi^{\prime}}{\partial x^{\prime}}, \\
& \omega^{\prime}=\frac{\partial^{2} \psi^{\prime}}{\partial x^{\prime 2}}+\frac{\partial^{2} \psi^{\prime}}{\partial y^{\prime 2}} .
\end{aligned}
$$

The vorticity and energy conservation equations are

$$
\begin{aligned}
& u_{x}^{\prime} \frac{\partial \omega^{\prime}}{\partial x^{\prime}}+u_{y}^{\prime} \frac{\partial \omega^{\prime}}{\partial y^{\prime}}=C_{1}^{\prime}\left(\frac{\partial^{2} \omega^{\prime}}{\partial x^{\prime 2}}+\frac{\partial^{2} \omega^{\prime}}{\partial y^{\prime 2}}\right)+C_{2}^{\prime} \frac{\partial T^{\prime}}{\partial x^{\prime}}-C_{3}^{\prime} \frac{\omega^{\prime}}{d^{\prime 2}}, \\
& u_{x}^{\prime} \frac{\partial T^{\prime}}{\partial x^{\prime}}+u_{y}^{\prime} \frac{\partial T^{\prime}}{\partial y^{\prime}}=\frac{\partial^{2} T^{\prime}}{\partial x^{\prime 2}}+\frac{\partial^{2} T^{\prime}}{\partial y^{\prime 2}} .
\end{aligned}
$$

The vorticity equation (5) involves three non-dimensional parameters: 


$$
C_{1}^{\prime}=\frac{v_{1}}{\kappa_{\mathrm{eff}}}, C_{2}^{\prime}=\frac{g \beta_{1} h^{3}\left(T_{1}-T_{0}\right)}{\kappa_{\mathrm{eff}}^{2}}, C_{3}^{\prime}=\frac{(1-\varepsilon)^{2}}{\varepsilon^{2}} \frac{15\left(\nu_{1}\right.}{\kappa_{\mathrm{eff}}},
$$

where $v, \mathrm{~g}$, and $\beta$ denote the kinematic viscosity, acceleration of gravity, and volume thermal expansion coefficient, respectively.

The third term of the right hand side of the Eq. (5) represents the frictional force between the liquid and solid surface. For the numerical simulations of the fluid flow in porous media, the term of frictional force $\mathbf{F}$ in momentum conservation equation is generally expressed in the Ergun's equation[8-11]:

$$
\mathbf{F}=\frac{150 v \mathbf{u}_{0}}{d^{2}} \frac{(1-\varepsilon)^{2}}{\varepsilon^{3}}+\frac{1.75 \mathbf{u}_{0}^{2}}{d} \frac{1-\varepsilon}{\varepsilon^{3}}
$$

where $\mathbf{u}_{0}$ denotes the volume averaged velocity vector defined in Ref. [12]. In the laminar region given by $\left(\left|\mathbf{u}_{0}\right| d / v\right) /(1-\varepsilon)<10$, the second term of the right hand side of Eq. (8), which represents flow inertia effects, is negligible. Then, the right hand side of Eq. (8) is reduced to only the first term, which is the Blake-Kozeny equation[13]. In the case when a flame spreads over a solid-liquid mixed layer, the liquid velocity is much smaller than that for the flame spread over a free-surface liquid pool[2], and the Blake-Kozeny equation can be adopted for prediction of the frictional force. By.taking rot of the term of Blake-Kozeny equation, i.e., the first term of right hand side of Eq. (8) and introducing the non-dimensional quantities defined above, the term of frictional force involved in Eq. (5) is obtained, as

$$
\frac{h^{2}}{\kappa_{\mathrm{eff}}} \operatorname{rot} \mathbf{F}=C_{3}^{\prime} \frac{\omega^{\prime}}{d^{\prime 2}}=\frac{(1-\varepsilon)^{2}}{\varepsilon^{2}} \frac{150 \mathrm{v}_{1}}{\kappa_{\mathrm{eff}}} \frac{\omega^{\prime}}{d^{\prime 2}} .
$$

In this case, as represented in Eq. (5), the frictional force which acts on the liquid in the reverse direction of its motion is proportional to the -2 nd power of the solid particle diameter.

\section{Boundary Conditions}

Based on the aforementioned assumptions, the following boundary conditions are adopted for the present numerical simulation.

$$
\begin{array}{ll}
y^{\prime}=0: & \frac{\partial T^{\prime}}{\partial y^{\prime}}=0 \text { for } x^{\prime}<0, T^{\prime}=1 \text { for } x^{\prime} \geq 0, \\
\psi^{\prime}=0, \omega^{\prime}=S^{\prime} \frac{\partial T^{\prime}}{\partial x^{\prime}}, \\
x^{\prime} \rightarrow \infty: & T^{\prime}=0, \psi^{\prime}=U^{\prime}, \omega^{\prime}=\frac{\partial^{2} \psi^{\prime}}{\partial y^{\prime 2}}, \\
& \frac{\partial T^{\prime}}{\partial x^{\prime}} \rightarrow 0, \frac{\partial \psi^{\prime}}{\partial x^{\prime}} \rightarrow 0, \frac{\partial \omega^{\prime}}{\partial x^{\prime}} \rightarrow 0,
\end{array}
$$


$x^{\prime} \rightarrow-\infty: \quad T^{\prime} \rightarrow 0, \psi^{\prime} \rightarrow-y^{\prime} U^{\prime}, \omega^{\prime} \rightarrow 0$.

$S^{\prime}$ involved in Eq. (10) is a non-dimensional parameter, which represents the magnitude of surface tension and is defined as follows:

$$
S^{\prime}=-\frac{h\left(T_{1}-T_{0}\right)}{\kappa_{\text {eff }} \mu_{1}} \frac{\mathrm{d} \sigma_{1}}{\mathrm{~d} T}
$$

where $\mu$ and $\sigma$ denote the fluid viscosity and surfacc tension, respectively.

\section{RESULTS AND DISCUSSION}

Basic equations(Eqs. (3)-(6)) are solved under the boundary conditions(Eqs. (10)-(13)) by a second-order finite difference method employing a grid made of $(100 \times 100)$ nodes. In this study, in order to compare the results with those of experiments performed by Takeno and Hirano[1, 2], the glass beads layer soaked with n-decane, whose depth and initial temperaturc are $4 \mathrm{~cm}$ and $23^{\circ} \mathrm{C}$ respectively, is considered as the solid-liquid mixed laycr. Table 1 summarizes parameters which are held or varied in this numerical study.

TABLE 1 Values of parameters adopted in the present study.

\begin{tabular}{|c|c|c|c|c|c|c|}
\hline & $C^{\prime}{ }_{1}$ & $C_{2}^{\prime}$ & $C_{3}^{\prime}$ & $S^{\prime}$ & $U^{\prime \text { a. }}$ & $d^{\prime}$ \\
\hline Case 1 & \multirow{7}{*}{$4.56 \times 10^{-1}$} & \multirow{4}{*}{$1.13 \times 10^{7}$} & \multirow{7}{*}{$5.59 \times 10^{2}$} & \multirow{5}{*}{$1.66 \times 10^{5}$} & 5.78 & 0.05 \\
\hline Case 2 & & & & & 6.82 & 0.075 \\
\hline Case 3 & & & & & 9.72 & 0.1 \\
\hline Case 4 & & & & & \multirow{3}{*}{15.2} & \multirow{3}{*}{0.125} \\
\hline Case 5 & & 0 & & & & \\
\hline Case 6 & & \multirow{2}{*}{$1.13 \times 10^{7}$} & & 0 & & \\
\hline Case 7 & & & & $1.66 \times 10^{5}$ & $2.31-11.5$ & 0.05 \\
\hline
\end{tabular}

a. Experimental data from Ref. [2] for Cases 1-6.

\section{Flow and Temperature Fields}

Typical flow and temperature fields predicted in this study are presented in Fig. 2, where the solid lines and dashed lines represent the streamlines and the isothermal lines, respectively. The values of $T^{\prime}$ corresponding with isothermal lines are $0.01,0.1,0.2$, and 0.5 , respectively. The leading flame edge is at $x^{\prime}=0$, and the liquid ahead of the leading flame edge moves from left to right. In Fig. 2(a), an eddy with a reverse surface flow appears near the surface. However, for a smaller value of $d^{\prime}$, the eddy becomes smaller as shown in Fig. 2(b). Further, it is seen that for a larger value of $d^{\prime}$, a high temperature region extends ahead of the leading flame edge, though that for a smaller $d^{\prime}$ scarcely extends toward upstream. Comparing the flow and temperature 


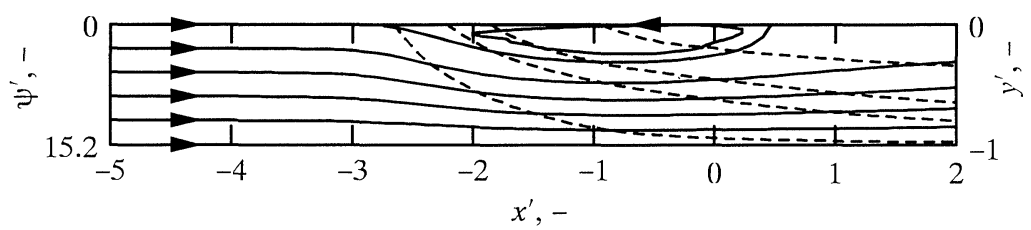

(a)

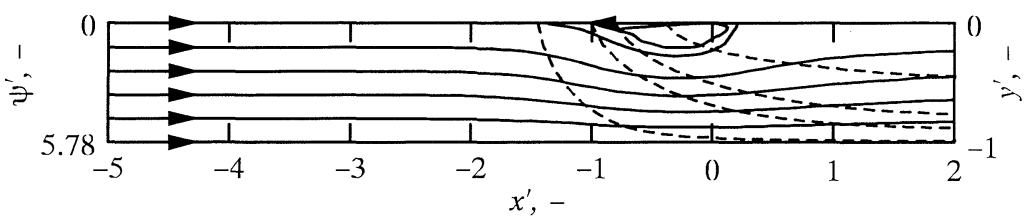

(b)

FIGURE 2 Typical predicted flow and temperature fields, (a) Case 4, $d^{\prime}=0.125$, (b) Case 1 , $d^{\prime}=0.05$, solid lines: stream lines, dashed lines: isothermal lines.

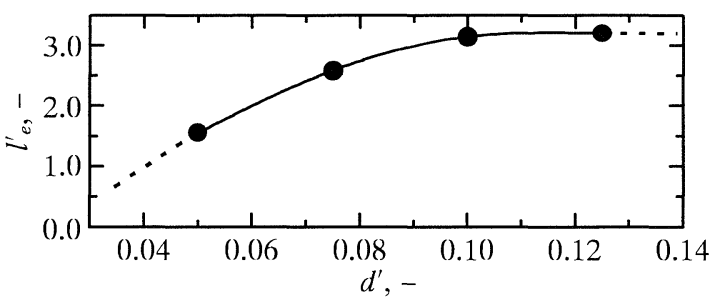

FIGURE 3 Non-dimensional eddy length along the layer surface for Cases 1-4.

fields presented in Fig. 2, the temperature distribution is found to be closely related to the liquid behavior.

The principal function of the eddy is to transport hot liquid from the region just beneath the leading flame edge to the region ahead of it. Therefore, the size of the eddy must be closely related to the flame spread phenomena. Figure 3 shows the non-dimensional surface length of the eddy along the layer surface, which is represented as $l_{\mathrm{e}}^{\prime}\left(=l_{\mathrm{c}} / h\right)$. Since the frictional force is proportional to $d^{\prime 2}$, the $l_{\mathrm{e}}^{\prime}$ increases as $d^{\prime}$ increases, and for a large $d^{\prime}$ the eddy size scems to approach its upper limit. This largest eddy size must be equal to that appearing at the flame spread over a free surface of the combustible liquid. 


\section{Heat Transfer Modes}

As aforementioned, for the initial temperature much lower than the flash point of the liquid, heat transfer through the solid-liquid mixed layer is needed for flame spread. Figure 4 shows non-dimensional integrated heat flux $Q^{\prime}\left(=Q /\left\{\lambda_{\text {eff }}\left(T_{1}-T_{0}\right)\right\}\right)$ at $x^{\prime}=0 . Q^{\prime}$ is divided into two parts in different heat transfer modes: conduction and convection. The integrated heat flux is evaluated as follows:

$$
\begin{aligned}
& Q_{\mathrm{cd}}^{\prime}=\int_{-1}^{0}\left[\frac{\partial T^{\prime}}{\partial x^{\prime}}\right]_{x^{\prime}=0} \mathrm{~d} y^{\prime} \equiv \int_{-1}^{0}\left(-q_{\mathrm{cd}}^{\prime}\right) \mathrm{d} y^{\prime}, \\
& Q_{\mathrm{cv}}^{\prime}=-\int_{-1}^{0}\left[\left(u_{x}^{\prime}-U^{\prime}\right) T^{\prime}\right]_{x^{\prime}=0} \mathrm{~d} y^{\prime} \equiv \int_{-1}^{0}\left(-q_{\mathrm{cv}}^{\prime}\right) \mathrm{d} y^{\prime},
\end{aligned}
$$

where the integrated heat flux in the direction ahead of the flame is positive. For values of $d^{\prime}$ smaller than 0.03 , the main mode of heat transfer is seen to be conduction. For this case, the model in the present simulation is not valid as mentioned in the section of "Formulation of Problem", so that we have not performed the numerical simulation. As $d^{\prime}$ increases the part of heat flux caused by conduction decreases and that by convection increases to be dominant at values of $d^{\prime}$ larger than 0.03 . This is the case to explore in the present study.

The distributions of non-dimensional heat flux $q_{\mathrm{cd}}^{\prime}$ and $q_{\mathrm{cv}}^{\prime}$ defined in Eq. (15) are shown in Fig. 5 for Case 1. Both conductive and convective heat transfer occurs mainly near the surface of the solid-liquid mixed layer, and the value of $q_{\text {cd }}^{\prime}$ is negative for any $y^{\prime}$. However, the value of $q_{\mathrm{cv}}^{\prime}$ is positive for $y^{\prime}<-0.35$. Also, the absolute value of $q_{\mathrm{cv}}^{\prime}$ is highly large near the layer surface, as is caused by the surface flow induced by the variation of the surface tension along the surface.

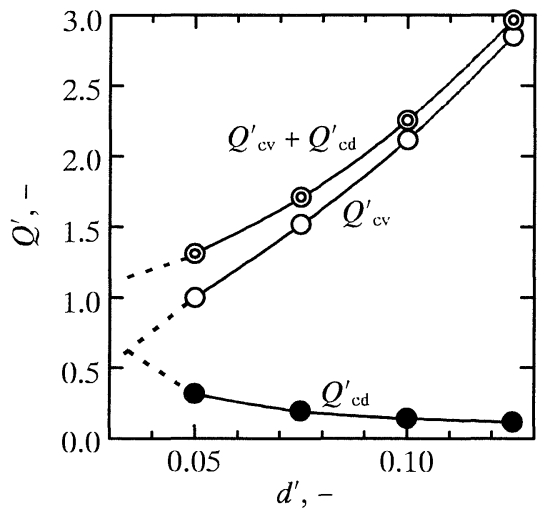

FIGURE 4 Non-dimensional integrated heat flux in direction of the flame at $x^{\prime}=0$ for Cases 1-4.

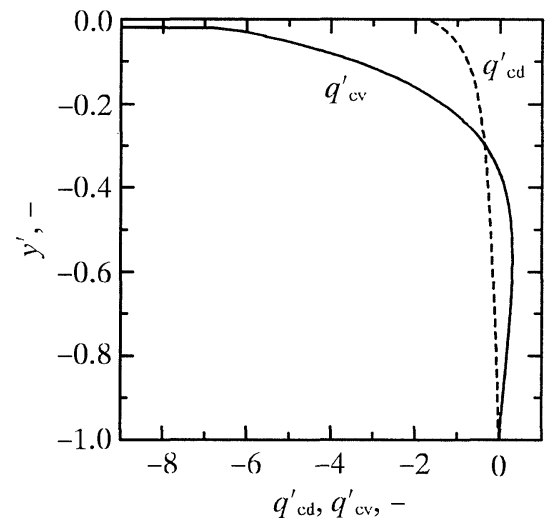

FIGURE 5 Distributions of non- dimensional heat flux at $x^{\prime}=0$ for Case 1 . 


\section{Driving Force of Liquid Motion}

The profiles of the horizontal component $u_{x}^{\prime}$ of the non-dimensional liquid velocity at $x^{\prime}=$ 0 are presented in Fig. 6 for Cases 4, 5 and 6 . For Case 4, the fast flow near the surface of the solid-liquid mixed layer is induced by the surface tension gradient along the surface. However, just below the surface, the velocity of the liquid is much smaller than that at the surface.

For the flame spread over a liquid pool, in general, the dominant force to induce a liquid flow in the region ahead of the leading flame edge is surface tension, and the effect of buoyancy is negligible. However, it was pointed out in Ref. [14] that buoyancy could play a significant role if the flame spread rate is slow enough. For the flame spread over a solid-liquid mixed layer, a flame spread rate is much smaller than that for the flame spread over a free-surface liquid pool. Therefore, it can be considered that buoyancy can play a significant role for the liquid motion in this case. As shown in Fig. 6, in the solid-liquid mixed layer, the horizontal velocity for Casc 5, which neglects the buoyancy, is quite different from that for Case 4. Except for a thin layer beneath the liquid surface, the liquid velocity in the solid-liquid mixed layer for Case 5 is much smaller than that for Case 4. On the other hand, for Case 6, which neglects the surface tension, the liquid velocity is nearly the same as that for Case 4 except for a smaller valuc at the surface. Due to frictional force between the liquid and solid surface, the depth of boundary laycr of surface tension flow becomes much smaller than that for flame spread over a frec-surface pool. This implies that buoyancy greatly influences the liquid motion in the solid-liquid mixed layer. Not only surface tension but also buoyancy contributes to the liquid motion for the flame spread over a liquid soaked in solid. Further, when $C_{2}^{\prime}$ is equal to zero, i.c., buoyancy is neglected(Case 5), the integrated heat flux $Q^{\prime}$ in the direction ahead of the flame becomcs less than half of the case when buoyancy is taken into consideration(Case 4), whilc $Q^{\prime}$ scarccly changes when $S^{\prime}=$ (), i.e., surface tension is neglected(Case 6). Consequently, the flame spread over a solid-liquid mixed layer is of different behavior from the flame spread over a liquid pool.

\section{Comparison with Experimental Results}

Figure 7 shows the temperature distributions for the case of $d^{\prime}=0.05$ at $y^{\prime}=-0.025$ obtained by the present numerical study and previous experiments[1]. It is seen that the predicted temperature distributions fairly well coincide with the experimental results.

Figure 8 shows the assumed heat flux from the gas phase for $x^{\prime} \geq 0$, which is represented by $q^{\prime}{ }_{11}$, given with a non-dimensional form as follows: 


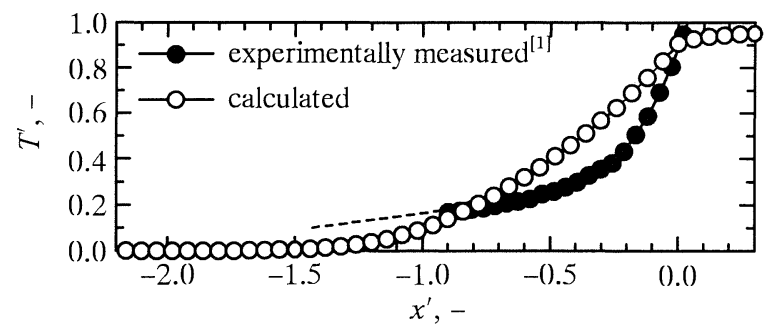

FIGURE 7 Non-dimensional temperature profiles ahead of the leading flame edge at $\mathrm{y}^{\prime}=-(0.025$ for Case 1 .

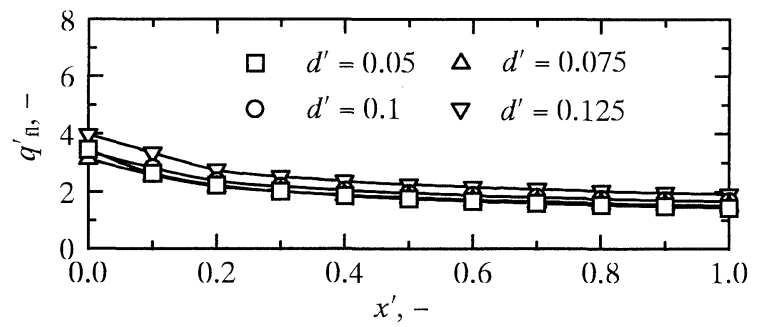

FIGURE 8 Non-dimensional assumed heat flux from the gas phase to the mixed layer beneath the flame for Cases 1-4.

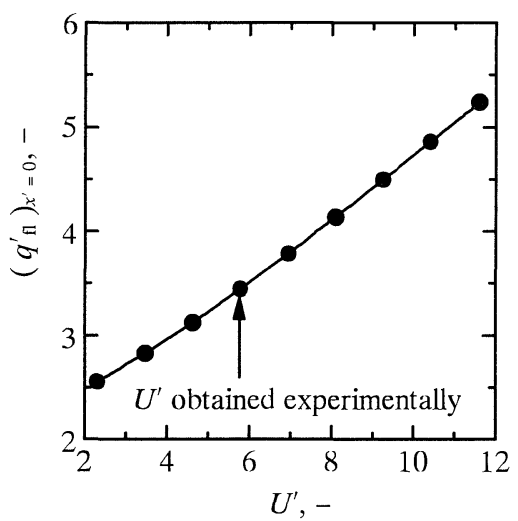

FIGURE 9 Variation of non-dimensional assumed heat flux for various nondimensional flame spread rates at $x^{\prime}=0$ for Case 7 . 
$q_{\mathrm{fl}}^{\prime}=\left|\frac{\partial T^{\prime}}{\partial y^{\prime}}\right|_{y^{\prime}=0}, q_{\mathrm{fl}}^{\prime}=\frac{h}{\lambda_{\text {eff }}\left(T_{1}-T_{0}\right)} q_{\mathrm{fl}}$.

In Fig. 8, $q_{\mathrm{fl}}^{\prime}$ at $x^{\prime}=0$ is nearly constant for all cases of $d^{\prime} \geq 0.05$. The value of this heat flux is 3 $<\left(q_{\mathrm{fl}}^{\prime}\right)_{x^{\prime}=0}<4\left(0.75 \mathrm{~W} / \mathrm{cm}^{2}<\left(q_{\mathrm{fl}}\right)_{x=0}<1 \mathrm{~W} / \mathrm{cm}^{2}\right)$, which is almost corresponding with the value obtained by previous experiments of flame spread over a n-propanol pool[15]. This result is attributable to the fact that the quenching distance and temperature of the leading flame edge should scarcely depend on the diameter of solid particles.

The value of $q_{\mathrm{fl}}^{\prime}$ at $x^{\prime}=0$ is changed by giving the various spread rates, which correspond to velocities at the far upstream boundary condition in the model shown in Fig. 1(b). Figure 9 shows the variation of the $q_{\mathrm{fl}}^{\prime}$ at $x^{\prime}=0$ for various $U^{\prime}$ s. As shown in Fig. 8, $q_{\mathrm{fl}}^{\prime}$ at $x^{\prime}=()$ is nearly constant for all cases $d^{\prime} \geq 0.05$. Therefore, it is implied that the spread rate $U^{\prime}$ for $d^{\prime} \geq 0.05$ can be approximately determined as an eigenvalue by selecting $U^{\prime}$ which satisfies the relation, $3<$ $\left[q_{\mathrm{fl}}^{\prime}\right]_{x^{\prime}=0}<4$.

A little difference can be seen between the temperature distributions near the surface measured experimentally and that predicted in this study(Fig. 7). This difference is considered to be attributable to the reasons as follows:

1) neglecting the thermal interaction between the gas phase and solid-liquid mixed layer at interface, such as:

heat transfer from gas phase to mixed layer and/or vice versa,

vaporization heat which must be large at the high temperature region,

2) neglecting the flow inertia effects in the friction term, so that the liquid velocity near the surface, where the liquid velocity is rather large, is considered to be overestimated. Then, the convective heat transfer, which is main mode of heat transfer, from the region just beneath the leading flame edge to the region ahead of it is overestimated.

\section{CONCLUSIONS}

A numerical simulation of liquid behavior and temperature distribution in a solid-liquid mixed layer during flame spread over it when the main mode of heat transfer to liquid ahead of the leading flame edge is convection. Main conclusions derived throughout this study are as follows:

1) Heat transfer to the layer ahead of the leading flame edge occurs mainly near the surface of the solid-liquid mixed layer.

2) Buoyancy greatly influences the liquid motion in the solid-liquid mixed layer ahead of the leading flame edge, so that not only surface tension but also buoyancy contributes to the liquid motion for the flame spread over a liquid soaked porous solid.

3) The predicted temperature distributions fairly well coincide with the experimental results.

4) The spread rate $U$ can be approximately determined as an eigenvalue by selecting $U$ which gives an appropriate value of the heat flux from gas phase to the solid-liquid mixed layer beneath the leading flame edge. 


\section{REFERENCES}

1. Takeno, K. and Hirano, T., "Flame spread over porous solids soaked with a combustible liquid”, Twenty-first Symposium (International) on Combustion, The Combustion Institute, 1986, pp.75-81.

2. Takeno, K. and Hirano, T., "Behavior of combustible liquid soaked in porous beds during flame spread", Twenty-second Symposium (International) on Combustion, The Combustion Institute, 1988, pp.1223-1230.

3. Torrance, K. E., "Subsurface flows preceding flame spread over a liquid fucl", Combustion Science and Technology 3, pp.133-143, 1971.

4. Torrance, K. E. and Mahajan, R. L., "Fire spread over liquid fucls: Liquid phasc parameters", Fifteenth Symposium (International) on Combustion, The Combustion Institute, 1974, pp.281-287.

5. Torrance, K. E. and Mahajan, R. L., "Surface tension flows induced by a moving thermal source", Combustion Science and Technology 10, pp.125-136, 1975.

6. Furuta, M., Humphrey, J. A. C. and Fernandez-Pello, A. C., "Prediction of flame spread hydrodynamics over liquid fuel", PhysicoChemical Hydrodynamics 6, pp.347-372, 1985.

7. Di Blasi, C., Crescitell, S. and Russo, G., "Model of pulsating flame spread across liquid fuels", Twenty-third Symposium (International) on Combustion, The Combustion Institute, 1990, pp.1669-1675.

8. Ergun, S., "Fluid flow through packed columns", Chemical Engincering Progress 48, pp.89-94, 1952.

9. Hong, J. T., Yamada, Y. and Tien, C. L., "Effects of non-Darcian and nonuniform porosity on vertical-plate natural convection in porous media”, ASME Journal of Heat Transfer 109, pp.356-362, 1987.

10. Beckermann, C., Ramadhyani, S. and Viskanta, R., "Natural convection flow and heat transfer between a fluid layer and a porous layer inside a rectangular cnclosure", ASME Journal of Heat Transfer 109, pp.363-370, 1987.

11. Vafai, K. and Sozen, M., "Analysis of energy and momentum transport for fluid flow through a porous bed", ASME Journal of Heat Transfer 112, pp.69()-699, 1990.

12. Vafai, D. and Tien, C. L., "Boundary and inertia effects on flow and heat transfer in porous media", International Journal of Heat and Mass Transfer 24, pp.195-203, 1981.

13. Bird, R. B., Stewart, W. E. and Lightfoot, E. N., Transport Phenomena, chapter 6, John Willey \& Sons, 1960.

14. Ross, H. D., "Ignition of and flame spread over laboratory-scale pools of pure liquid fuels", Progress in Energy and Combustion Sciences 20, pp.17-63, 1994.

15. Ito, A., Masuda, D. and Saito, K., "A study of flame spread over alcohls using holographic interferometry", Combustion and Flame 83, pp.375-389, 1991. 